\title{
Reduced GABAergic neuronal activity in zona incerta causes neuropathic pain in a rat sciatic nerve chronic constriction injury model
}

This article was published in the following Dove Press journal:

Journal of Pain Research

II May 2017

Number of times this article has been viewed

\author{
Hyeong Cheol Moon' \\ Young Seok Park ${ }^{1,2}$ \\ 'Department of Medical \\ Neuroscience, ${ }^{2}$ Department \\ of Neurosurgery, Neurofuture \\ Laboratory, College of Medicine, \\ Chungbuk National University \\ Hospital, Cheongju-si, Chungbuk, \\ Republic of Korea
}

Correspondence: Young Seok Park Department of Neurosurgery, Neurofuture Laboratory, College of Medicine, Chungbuk National University Hospital, 4I 0 SungBong-Ro Heungdeok-gu, Cheongju-si, Chungbuk, Republic of Korea

Tel +82432696080

Fax +82432731614

Email youngseokparkmd@gmail.com
Purpose: The zona incerta (ZI) is below the ventral tier of the thalamus and has a strong influence selectively in higher-order thalamic relays. Although neuropathic pain has been suggested to result from reduced gamma-aminobutyric acid (GABA) and GABAergic signaling in the ZI, the mechanisms remain unclear. Here, the role of GABA and GABAergic signaling was investigated in the ZI in neuropathic pain using sciatic nerve chronic constriction injury (CCI) rats. Materials and methods: Single-unit neuronal activity was recorded, and microdialysis was performed in the ZI of CCI rats and sham-treated rats in vivo. This study also compared ZI neuronal activity after treatment with saline, the $\mathrm{GABA}_{\mathrm{A}}$ receptor agonist (muscimol), or the $\mathrm{GABA}_{\mathrm{A}}$ receptor antagonist (bicuculline).

Results and conclusion: $\mathrm{CCI}$ rats exhibited hypersensitivity to pain as evidenced by decreased hind paw withdrawal threshold and latency. CCI rats also showed reduced GABA level and decreased neuronal activity in the ZI compared with sham-treated rats. Treatment with $\mathrm{GABA}_{\mathrm{A}}$ receptor agonist, but not $\mathrm{GABA}_{\mathrm{A}}$ receptor antagonist, ameliorated pain hypersensitivity and increased the firing rate (spikes/s) of ZI neurons in CCI rats.

Keywords: sciatic nerve injury, chronic pain, neuropathic pain, zona incerta, neural cell recording, GABA

\section{Introduction}

Neuropathic pain commonly accompanies a variety of conditions, including peripheral nerve injury (eg, resulting from postsurgical pain or radiation), central nervous system injury (eg, multiple sclerosis, spinal cord injury), viral infections (eg, postherpetic neuralgia), tumors, and metabolic disorders, such as diabetes mellitus. ${ }^{1-5}$ Severity and duration are often greater than that of other types of chronic pain, ${ }^{6}$ and $5 \%$ of adults with neuropathic pain suffer debilitating pain despite analgesic pharmaceutical therapy. ${ }^{7,8}$ Thus, neuropathic pain is one of the most discouraging clinical findings, not controllable with standard pain medications, and resistant to many drugs. Chronic neuropathic pain resulting from peripheral nerve damage is a significant clinical problem that often proves refractory to current treatments. ${ }^{2}$ Patients in neuropathic pain suffer from shooting and burning pain, electric shock-like pain, tingling and numbness, dysesthesia, paresthesia, allodynia, and hyperalgesia. ${ }^{9-11}$ Chronic constriction injury (CCI) of the sciatic nerve is commonly used to generate rat models of neuropathic pain. Although the cause of CCI-induced pain is unknown, chronic neuropathic pain is thought to result from abnormal zona incerta (ZI)-mediated suppressed inhibition in the thalamus, ${ }^{12}$ and this incerto-thalamic inhibition of the posterior thalamic nucleus is mediated by gamma-aminobutyric acid (GABA).${ }^{13-15}$ Efforts to identify the mechanisms 
by which GABAergic transmission modulates states of pain are complicated by the fact that levels of GABAergic transmission vary by brain region. ${ }^{15-18}$

Many previous studies report links between the ZI and chronic pain. ${ }^{19,20}$ The ZI is a GABAergic nucleus located in the ventral thalamus, which is a subdivision of the diencephalon, and most ZI neurons are GABAergic. ${ }^{21,22}$ Interneuron has inhibitory pathways, such as GABAergic pathways, which contribute to the balance between excitatory and inhibitory tone in ZI synaptic transmission. Recently, Masri et al described a novel system for the regulation of nociceptive processing in the thalamus in which the ZI, which is one of the most cytoarchitectonically and neurochemically diverse cell groups within the thalamus, inhibits the flow of nociceptive and somatosensory information in the posterior thalamus. ${ }^{12,23}$ The ZI regulates both spontaneous and evoked activity in the posterior thalamus. ${ }^{24,25}$ $\mathrm{ZI}$ inactivation results in reduced response latency in a subset of posterior thalamic nucleus. ${ }^{12,24}$ The ZI contains pathways (primary and secondary) that orchestrate the somatosensory system and the interoceptive system. ${ }^{21}$

To test GABA and GABAergic signaling, the present study examined alterations in GABA concentration and GABAergic ZI neuronal activity in vivo using a rat model of sciatic nerve CCI. Sciatic nerve CCI in rats results in relatively consistent neuropathic pain behavior that mimics neuropathic pain caused by peripheral nerve injury in humans. Pain-related behavior and neuronal activity after infusion of $\mathrm{GABA}_{\mathrm{A}}$ receptor agonist and antagonist into the $\mathrm{ZI}$ were evaluated.

\section{Materials and methods}

\section{Experimental animals and design}

All animal experiments were performed in accordance with the approved national guidelines established by the ethics review committee of Chungbuk National University for Animal Experiments. Aseptic surgical procedures were used according to the guidelines of the International Association for the Study of Pain. Sterile animal surgical equipment, gloves, and aseptic drapes and swabs were used. Skin was prepared with $70 \%$ ethanol, and fur was shaved in the surgical area, which was then covered with a sterile drape. Sprague Dawley male rats (250-300 g; Daehan Biolink, Eumseong, South Korea) were maintained in accordance with National Institutes of Health guidelines on animal welfare under standard housing conditions (12:12 hour light-dark cycle) and given laboratory food pellets and water ad libitum. Rats were housed in separate cages with flat paper bedding to prevent choking in this randomized, double-blind, controlled animal trial. The timeline of the experiments is shown in Figure 1. The sample size was determined using PASS 14 software (NCSS Inc, Kaysville, UT, USA; alpha $=0.05$, power $=0.8$ ).

\section{$\mathrm{CCl}$ surgery}

CCI $(n=20)$ and sham-treated $(n=10)$ rats were used in this study. All animals were assigned a group designation on the same day. Cages were randomly selected from the pool of all cages and assigned to groups. Cages were given a temporary numerical designation, and rats were assigned numbers by the registrar. At surgery, all animals received the same anesthesia, prep, sedation, and preliminary surgical procedures. Postsurgical blinding was then carried out. Animals were removed from the cage for this study and given a permanent designation by the registrar.

\section{Behavioral testing}

Mechanical hyperalgesia was measured with a Dynamic Plantar Aesthesiometer (model 37450; Ugo Basile, Varese, Italy). Rats $(n=30)$ were placed in a Plexiglas cage $(20 \times 20 \times 14 \mathrm{~cm})$ with a grid bottom and habituated for at least $40 \mathrm{~min}$. Mechanical stimuli were generated by placing continuously increasing pressure $(5 \mathrm{~g} / \mathrm{s})$ on the plantar region of the paws ipsilateral and contralateral to the CCI lesion. Hind paw withdrawal threshold (PWT) and paw withdrawal latency (PWL) were measured after injection of $2 \mu \mathrm{L}$ of either saline,

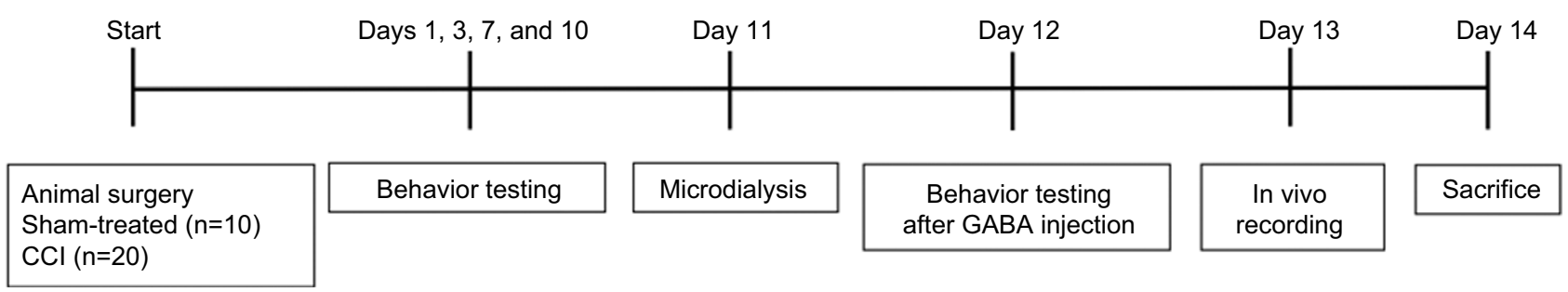

Figure I Flowchart illustrating the study procedure.

Abbreviations: $\mathrm{CCl}$, chronic constriction injury; GABA, gamma-aminobutyric acid. 
$\mathrm{GABA}_{\mathrm{A}}$ agonist muscimol $(0.5 \mathrm{mmol} / \mu \mathrm{L}[0.28525 \mu \mathrm{g}]$ or $5 \mathrm{mmol} / \mu \mathrm{L}[2.8525 \mu \mathrm{g}])$, or the $\mathrm{GABA}_{\mathrm{A}}$ antagonist bicuculline $(0.5 \mu \mathrm{mol} / \mu \mathrm{L}[0.0009185 \mu \mathrm{g}]$ or $5 \mu \mathrm{mol} / \mu \mathrm{L}[0.009185$ $\mu \mathrm{g}])$. Low- and high-concentration doses were determined based on previous reports. ${ }^{26-28}$ Behavioral tests were carried out at least 2 hours after drug administration. Each value represents the mean of three independent measurements.

\section{Implantation of guide cannula}

To perform microdialysis and administer GABAergic drugs, a guide cannula was implanted 4 days before experiments. A longitudinal incision was made along the midline of the skull to expose bregma and lambda. A microguide tube (Eicom, Kyoto, Japan) for recording and drug infusion was fixed in place using two bone screws and acrylic resin (Ortho-Jet; Lang Dental, Wheeling, IL, USA). Under $15 \mathrm{mg}$ / $\mathrm{kg}$ tiletamine/zolazepam and $9 \mathrm{mg} / \mathrm{kg}$ xylazine anesthesia, a stainless steel guide cannula (outer diameter, $0.5 \mathrm{~mm}$; AG-8; Eicom) was implanted stereotaxically in the ZI at the following coordinates: $3.5 \mathrm{~mm}$ anterior to bregma, $2.8 \mathrm{~mm}$ lateral to the midline, and $6.8 \mathrm{~mm}$ below the surface of the brain according to the atlas of Paxinos and Watson. ${ }^{29}$ After implantation of the guide cannula, a dummy cannula (outer diameter, $0.5 \mathrm{~mm}$ ) was carefully inserted.

\section{In vivo microdialysis}

On the day before microdialysis, the inner stylet was replaced with a dialysis probe with a $1.0 \mathrm{~mm}$ long semipermeable membrane (outer diameter, $0.31 \mathrm{~mm}$; A-I-8-02; Eicom). A two-channel fluid swivel device (SSU-20, Eicom) was connected to the inlet and outlet of the probe, and artificial cerebrospinal fluid (147 mM NaCl, $4 \mathrm{mM} \mathrm{KCl}, 1.2 \mathrm{mM}$ $\mathrm{CaCl}_{2}$, and $0.9 \mathrm{mM} \mathrm{MgCl}_{2}$ ) was infused through the probe at a rate of $1 \mu \mathrm{L} / \mathrm{min}$ using an infusion pump. Dialysis was performed under anesthetized, unrestrained conditions. ${ }^{30-33}$ After an overnight equilibrium period, $36 \mu \mathrm{L}$ dialysate was collected every $30 \mathrm{~min}$ in vials containing an equal volume $(36 \mu \mathrm{L})$ of acetic acid solution $(40 \mathrm{mM})$ with ethylenediaminetetraacetic acid $(200 \mu \mathrm{M})$. Samples were stored at $-70^{\circ} \mathrm{C}$ until assay. GABA, glutamic acid, and serotonin were measured in sham-treated $(n=10)$ and CCI $(n=10)$ rats.

\section{Microinjection procedures}

Drug or saline was microinjected into the ZI using a microdialysis probe protected by a system of telescoping steel tubes. ${ }^{34}$ The assembly was inserted into the guide cannula immediately before microinjection, and the needle was advanced to protrude $3 \mathrm{~mm}$ beyond the guide cannula tip.
Because the area of the $\mathrm{ZI}\left(\sim 3 \mathrm{~mm}^{2}\right)$ is larger than that of the anterior pretectal nucleus $\left(\sim 2 \mathrm{~mm}^{2}\right)$, a rate of $0.25 \mu \mathrm{L} /$ min was chosen for each injection. Solution was delivered at this rate for $2 \mathrm{~min}$, and the needle was removed $20 \mathrm{~s}$ after completion of the procedure.

\section{In vivo extracellular recordings}

At least 10 days after sciatic nerve injury, sham-treated and CCI rats ( $\mathrm{n}=6$ per group) were anesthetized with $15 \mathrm{mg} / \mathrm{kg}$ tiletamine/zolazepam and $9 \mathrm{mg} / \mathrm{kg}$ xylazine. Extracellular recordings were obtained from the ZI through quartz-insulated carbon electrodes (Kation Scientific, Minneapolis, MN, USA). The study showed that waveforms were digitized (40 $\mathrm{kHz}$ ) by a Digital Lynx SX data acquisition system (Neuralynx Inc., Bozeman, MT, USA), and units were sorted offline with Neuralynx SpikeSort 3D software using dual thresholds and principle component analysis. Firing rates (spikes/s) and peristimulus time histograms (PSTHs) were generated with NeuroExplorer software (Neuralynx) to confirm that recordings were obtained from single units. ${ }^{35}$

\section{Neurotransmitter analysis}

Liquid chromatography-mass spectrometry (LC-MS) was used to detect GABA, glutamate, and serotonin. Chromatographic analysis was performed on an Agilent 1100 high-performance liquid chromatography (HPLC) system (Agilent Technologies, Santa Clara, CA, USA) comprising a G1322A degasser, a G1311A quaternary pump, a G1313A well-plate autosampler, and a G1316A thermostated column compartment. A G1946D mass spectrometer (Agilent Technologies) prepared with an electrospray source interface was used for MS detection. Data acquisition and analysis were performed using Agilent ChemStation for LC/MS detection (version B.02.01). Chromatographic separation was achieved on an Agilent XDB-C18 column (3.0 mm $\times 50$ $\mathrm{mm}$; id, $1.8 \mu \mathrm{m})$ and eluted with a mobile phase of acetonitrile:0.1\% formic acid aqueous solution $(24: 76, \mathrm{v} / \mathrm{v})$ at a flow rate of $0.3 \mathrm{~mL} / \mathrm{min}$. The column temperature was maintained at $25^{\circ} \mathrm{C}$, the autosampler at $4^{\circ} \mathrm{C}$, and the volume was $5 \mu \mathrm{L}$. The analysis time was $4.5 \mathrm{~min}$ per sample. The HPLC system was connected to the mass spectrometer via an electrospray ionization (ESI) interface. The mass conditions of ESI were optimized in the negative ion detection mode as follows: capillary $3,500 \mathrm{~V}$, nebulizer $40 \mathrm{psi}$, drying gas $8 \mathrm{~L} / \mathrm{min}$, gas temperature $350^{\circ} \mathrm{C}$, and fragmentor $80 \mathrm{~V}$. Selected ion monitoring was used, and the fragmentation transitions were m/z 511.1 for curculigoside and m/z 579.1 for naringin. 


\section{Histological verification of electrode and probe placement}

After completion of the experiments, the electrode and probe sites were verified postmortem. Electrolytic lesions were made before cardiac perfusion by passing a $0.05 \mathrm{~mA}$ current through the recording electrode for $10 \mathrm{~s}$. Rats were deeply anesthetized with tiletamine/zolazepam and perfused through the heart with $4 \%$ paraformaldehyde in $0.1 \mathrm{M}$ phosphate-buffered saline. Rats were then transcardially perfused with saline followed by $10 \%$ formalin in saline. Brains were extracted and further fixed in $10 \%$ formalin for 24 hours before sectioning. Coronal sections $(5 \mu \mathrm{m})$ were cut through the thalamic formation using a microtome cryostat (Microm, Walldorf, Germany) and target sites in the brains were stained with neutral red. Electrode and probe sites were located under a light microscope based on the atlas of Paxinos and Watson. ${ }^{29}$

\section{Statistical analysis}

Analysis was carried out using two-way analysis of variance (ANOVA) of behavior using the statistical software program Prism 7.0 (GraphPad Software, Inc., La Jolla, CA, USA) and unpaired $t$-test of microdialysis concentration. To investigate the effects of drugs after injection, one-way ANOVA test of behavior and neuronal activity recording were performed each time. For all experiments, $P<0.05$ was considered significant. Data represent the mean \pm standard deviation (SD) for behavioral tests and mean \pm standard error of the mean for neural signal and biochemical analysis.

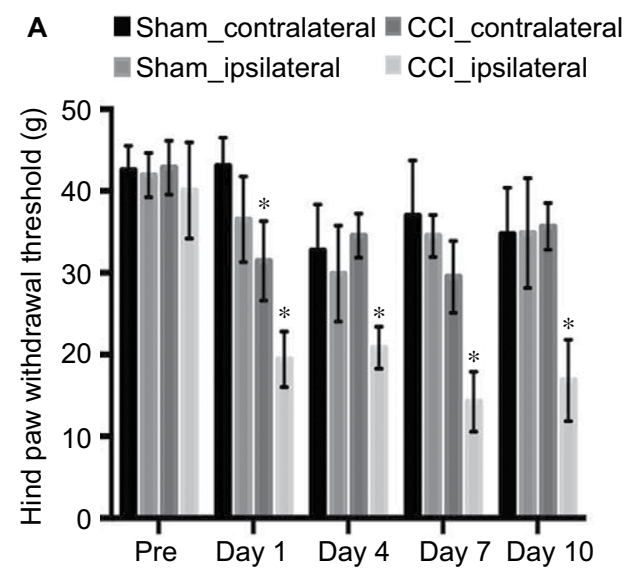

\section{Results}

\section{Behavioral confirmation of hypersensitivity to pain}

To assess sensitivity to neuropathic pain in CCI $(n=20)$ and sham-treated $(n=10)$ rats, behavioral assays were carried out to find PWT and PWL. PWT of the ipsilateral hind paw in CCI rats decreased significantly, from 35.66 \pm 2.85 (mean \pm $\mathrm{SD})$ to $16.84 \pm 4.98 \mathrm{~g}$ (repeated two-way ANOVA, $F=51.21$, $P=0.0001)$ compared to the contralateral hind paw and shamtreated animals over 10 days post-surgery (Figure 2A). Consistent with previous reports, ${ }^{36-38}$ animals with sciatic nerve injury showed a reduction in PWT in response to mechanical stimulation after surgery.

It was also found that PWL of the ipsilateral hind paw of CCI rats decreased significantly, from $6.81 \pm 1.57$ to $3.31 \pm 1.01 \mathrm{~s}$ (repeated two-way ANOVA, $F=65.99, P=0.0001$ ) compared with the contralateral hind paw and sham-treated animals over 10 days after surgery (Figure 2B). Importantly, PWT and PWL of CCI rats remained significantly lower than those of sham-treated rats over the same time period. Sham-treated rats showed no differences in PWT and PWL over this time period.

\section{In vivo microdialysis of the $\mathrm{ZI}$}

To assess changes in neurotransmitter levels, microdialysis probes were implanted in the ZI of CCI $(n=10)$ and sham-treated rats $(n=10)$. CCI rats showed significantly lower GABA levels $(2.31 \pm 0.61 \mu \mathrm{g} / \mathrm{L})$ than sham-treated rats $(5.38 \pm 0.43 \mu \mathrm{g} / \mathrm{L}$; unpaired two-tailed $t$-test, $P<0.05$;

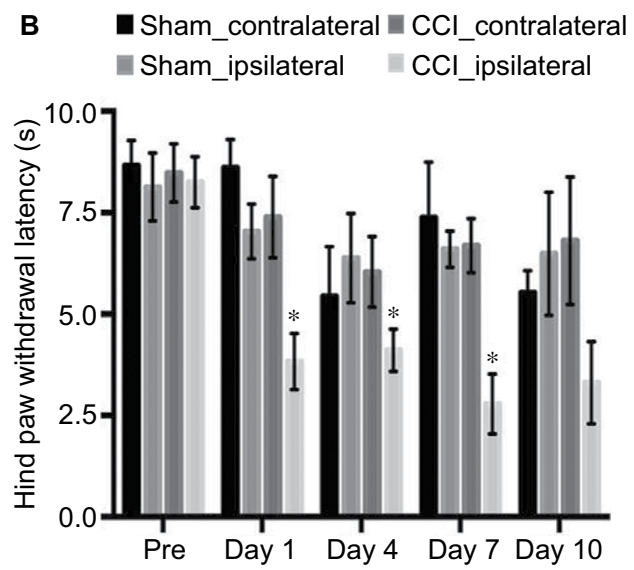

Figure 2 Change in PWTs on days I, 4, 7, and 10 after $\mathrm{CCl}$ and sham treatment.

Notes: PWTs were assessed by plantar test. (A) Mechanical withdrawal thresholds in plantar surface of hind paw decrease over time, and hyperalgesia develops ipsilaterally after sciatic nerve injury. (B) Mechanical withdrawal latency also decreased over time. All data points represent the mean \pm SD. Two-way ANOVA was used to assess statistical differences; ${ }^{*}<0.05$. $\mathrm{CCl}$ rats responded with significantly shorter latencies to mechanical stimulation of the plantar area of the ipsilateral hind paw than did shamtreated rats. Sham-treated $(\mathrm{n}=10) ; \mathrm{CCl}(\mathrm{n}=20)$.

Abbreviations: ANOVA, analysis of variance; $\mathrm{CCl}$, chronic constriction injury; PWTs, paw withdrawal thresholds; SD, standard deviation. 
Figure 3). CCI and sham-treated rats showed similar levels of glutamic acid. Furthermore, CCI rats showed significantly lower serotonin levels $(0.25 \pm 0.07 \mu \mathrm{g} / \mathrm{L})$ than sham-treated rats $(0.46 \pm 0.05 \mu \mathrm{g} / \mathrm{L}, P<0.05)$.

\section{Effects of GABAergic injection in $\mathrm{ZI}$ on withdrawal thresholds}

To investigate the contribution of GABA in the ZI to pain control, a guide cannula was implanted into the $\mathrm{ZI}$ of $\mathrm{CCI}$ $(n=10)$ and sham-treated $(n=10)$ rats and $2 \mu \mathrm{L}$ of either saline, muscimol $(0.5$ or $5 \mathrm{mmol} / \mu \mathrm{L})$, or bicuculline $(0.5$ or $5 \mu \mathrm{mol} / \mu \mathrm{L}$ ) was microinjected using a microdialysis probe at a rate of $0.25 \mu \mathrm{L}$ per minute. The higher dose of muscimol injection increased PWT (one-way ANOVA, $F=3.86$, $P<0.05$ ) and PWL (one-way ANOVA, $F=3.60, P<0.05$ ) of the ipsilateral hind paw, whereas neither dose of bicuculline had an effect on PWT or PWL (Figure 4A and B).

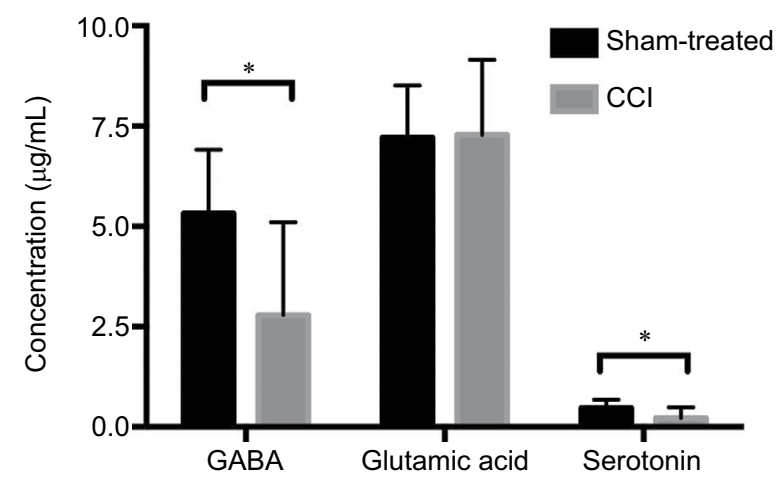

Figure 3 Analysis of neurotransmitter concentrations in the Zl using microdialysis in vivo.

Notes: $\mathrm{CCl}$ rats $(n=10)$ showed lower GABA concentrations than sham-treated rats $(n=10)$. Two-tailed $t$-test was used to compare GABA levels. $* P<0.05$.

Abbreviations: $\mathrm{CCl}$, chronic constriction injury; GABA, gamma-aminobutyric acid; $\mathrm{ZI}$, zona incerta.

\section{Neuronal activity in Zl following sciatic nerve injury}

To evaluate the effect of sciatic nerve injury on ZI neuronal output, the study recorded neuronal activity in the ZI of CCI $(n=6)$ and sham-treated $(n=6)$ rats. The target $Z I$ is shown in the schematic diagram of a coronal section (Figure 5A). The average firing rate of ZI neurons was lower in CCI than in sham-treated rats (Figure 5B). Furthermore, the spontaneous firing rate was significantly lower in CCI rats $(5.80 \pm 0.12$ spikes/s) than in sham-treated rats $(7.73 \pm 0.15$ spikes/s, unpaired $t$-test, $P<0.001)$. To analyze the effect of GABA modulation of the ZI on neuronal activity, sham-treated and CCI rats treated with muscimol or bicuculline were studied and spontaneous firing rates were measured for $30 \mathrm{~min}$. Firing rate in sham-treated rats increased significantly after muscimol and bicuculline injection compared to control saline injection, based on one-way ANOVA each time (Figure 5C, $P<0.05$; Table S1). Firing rates increased above baseline in CCI rats after injection of muscimol, whereas injection of bicuculline did not affect the firing rate, based on one-way ANOVA (Figure 5D, $P<0.05$; Table $\mathrm{S} 1$ ). Overall changes in firing rates of CCI rats after GABAergic drug treatment, as demonstrated by PSTHs, are shown in Figure 5E and F. Neuronal cell recording was monitored in 12 rats.

\section{Discussion}

This study was designed to examine if neuropathic pain was related by GABAergic neuronal activity of neurotransmitters in the ZI. Studies have shown that neuronal activity in the ZI is significantly lower in the CCI of sciatic nerve rat models compared to sham-controlled rats. In addition, treatment

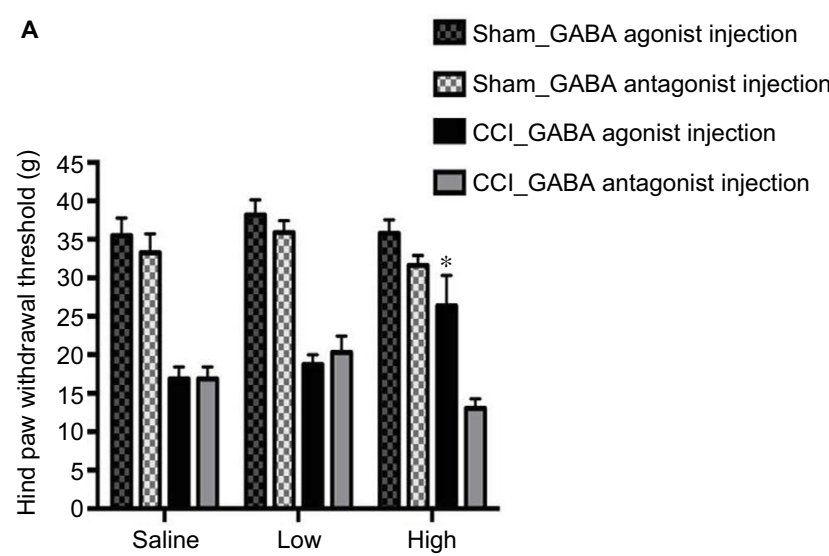

\section{B}

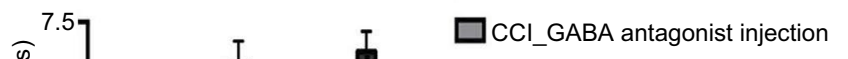

Figure 4 Changes in mechanical hyperalgesia induced by agonist, antagonist, or saline injection in sham-treated and $\mathrm{CCl}$ rats.

Notes: (A) Hind PWTs change from GABAergic concentrations in the ipsilateral developed hyperalgesia. (B) Hind PWL increased after injection of a high concentration of GABA agonist. One-way ANOVA was used to compare the effect of injection in each group. Sham-treated $(n=10) ; C C l(n=10)$. $* P<0.05$

Abbreviations: ANOVA, analysis of variance; $\mathrm{CCl}$, chronic constriction injury; GABA, gamma-aminobutyric acid; PWL, paw withdrawal latency; PWTs, paw withdrawal thresholds. 
A

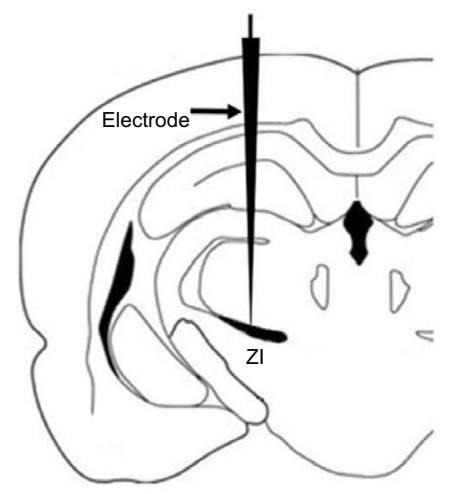

D

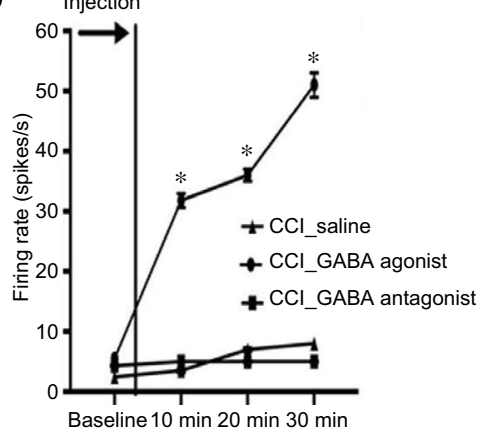

B

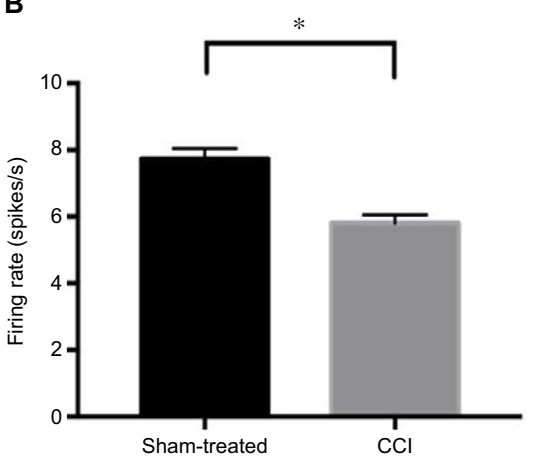

E

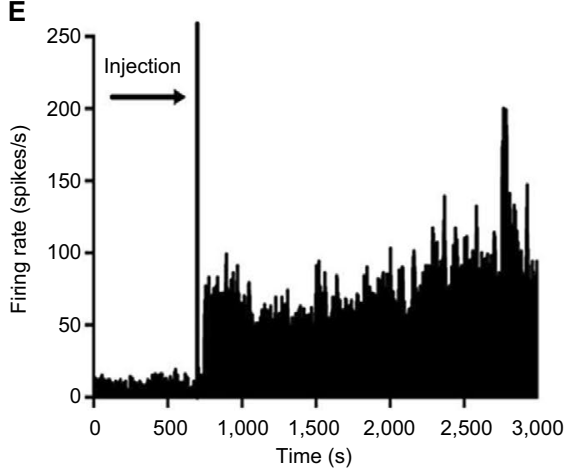

C

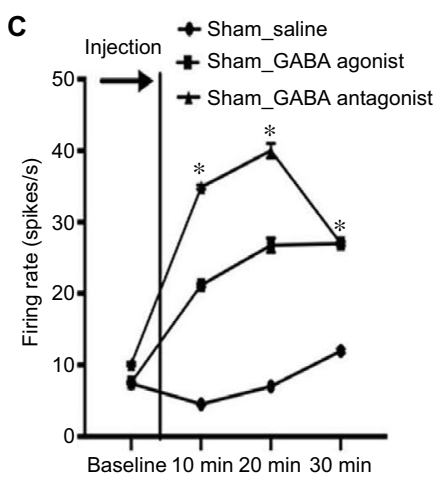

F

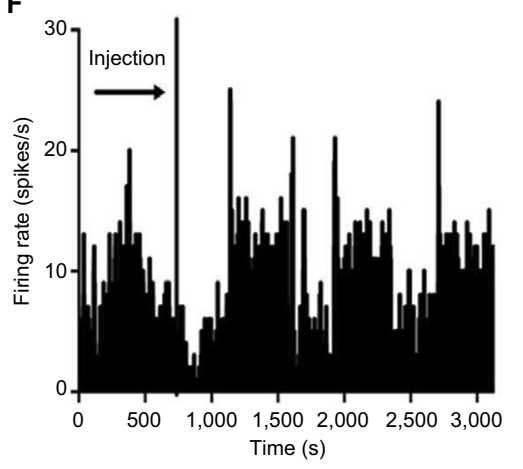

Figure 5 Spontaneous neuronal activity in the $\mathrm{Zl}$ in sham-treated and $\mathrm{CCl}$ rats.

Notes: (A) $\mathrm{Zl}$ is shown in the schematic diagram of a coronal section. (B) Overall thalamic neuronal activity in sham-treated and $\mathrm{CCl}$ rats. (C) Injection of high concentrations of $\mathrm{GABA}_{A}$ agonist and antagonist into the $\mathrm{Zl}$ changed the firing rate (spikes/s) for 30 min in sham-treated animals. (D) Firing rate increased after injection of $\mathrm{GABA}$ agonist in $\mathrm{CCl}$ rats. PSTH shows firing rate as time progresses after injection of $\mathrm{GABA}_{\mathrm{A}}$ agonist $(E)$ and $\mathrm{GABA}$ antagonist $(F)$ in $C C l$ rats. In total, 12 rats were used in the current analysis. One-way ANOVA was used to compare each data point with saline-baseline. $* P<0.05$. Sham-treated $(n=6)$; $C C l(n=6)$.

Abbreviations: ANOVA, analysis of variance; $\mathrm{CCl}$, chronic constriction injury; GABA, gamma-aminobutyric acid; PSTH, peristimulus time histogram; Zl, zona incerta.

with the GABA Agonist ameliorated the decreased PWT and PWL in the sciatic nerve injury rat model and increased the firing rate in the $\mathrm{ZI}$. All these data suggest that suppressed GABA neuronal activity is a primary cause of neuropathic pain following $\mathrm{CCI}$ in the rat model.

Previous studies have shown neural circuits from the ZI to thalamic relays, including the intralaminar nuclei. The ZI projects into a large number of thalamic nuclei ${ }^{39}$ and exerts feed-forward and tonic inhibition on the thalamus. ${ }^{24,25}$ Decreased neuronal activity has been previously demonstrated in the ZI in a spinal cord injury-induced neuropathic pain rat model. ${ }^{13}$ The loss of ZI activity likewise correlates with a pathological increase in neuronal activity in the posterior thalamus, a somatosensory thalamic nucleus critical for processing nociceptive information. ${ }^{12}$ Modulation of ZI firing rate and synaptic GABA concentration has been shown to be an effective means of regulating the incerto-thalamic circuit in a computational study. ${ }^{15}$ It has been shown that this GABAergic modulation in the ZI alleviates neuropathic pain in vivo.

These pathways are inhibitory (GABAergic) and are likely to serve as modulators of thalamic transmission. ${ }^{23}$ The
ZI receives topographically organized projections from the deep layers of the superior colliculus, the sensory trigeminal nuclear complex, and the dorsal column nuclei and contains primarily GABAergic neurons that project to the superior colliculus and the higher-order thalamic nuclei, such as the posterior and anterior pretectal nuclei and contralateral ZI. ${ }^{40}$ The ZI neurons innervate various brain regions, including the dorsal thalamus and cerebral cortex; therefore, stimulation of GABAergic incerta neurons using ultrahigh frequency stimulation or muscimol infusion may result in a transient disinhibition of incerta-projecting brain areas. ${ }^{41}$ Multiple active zones of ZI terminals indicate a powerful influence on the firing properties of thalamic neurons, which is conveyed to multiple cortical areas via relay cells, which have widespread projections to the neocortex. GABAergic terminals from the ZI selectively innervate the proximal dendrites of relay neurons, mainly in higher-order nuclei of the thalamus. ${ }^{42}$ The ZI forms a primal center of the diencephalon, a horizontally elongated region wedged just below the ventral tier of the thalamus, and generates direct responses (visceral, arousal, attention, and/or posture-locomotion) to a given sensory (somatic and/or visceral) stimulus. ${ }^{21,39,43}$ 
Sciatic nerve CCI is a widely used peripheral chronic neuropathic pain model. It is relatively simple to perform and produces robust and stable pain hypersensitivity for at least 1 month after injury. Following sciatic nerve CCI, rats exhibit abnormal posturing of the injured hind paw (ie, toes held together and paw plantar-flexed and everted), repeated shaking of the paw, and guarding and licking of the paw, suggesting the presence of spontaneous pain. ${ }^{10} \mathrm{CCI}$ produces maladaptive synaptic circuits in the spinal dorsal horn that individually or synergistically contribute to neuronal hyperexcitability. This neuronal hyperexcitability or central sensitization is characterized by enhanced spontaneous or evoked neuronal responses to external stimuli applied to peripheral receptive fields, lowered thresholds for activation, increased peripheral receptive field size, and increased afterdischarge activity. ${ }^{446}$

As discussed, the ZI neurons are innervated by the activity of thalamic nuclei. According to a previous study, neuropathic pain is associated with a reduction in ZI neurons. Decreased GABAergic phasic inhibitory transmission is thought to be responsible for the development of neuropathic pain. ${ }^{47}$ Therefore, the mechanisms underlying CCI of sciatic nerve are under investigation, in which CCI lesions show suppression of the gradual apoptosis of GABAergic neurons in the ZI. Microdialysis was used to demonstrate that inactivation of the ZI neurons is related to the suppression of GABAergic neurons, and neuronal activity in the $\mathrm{ZI}$ is controlled by GABA treatment. Lower levels of GABA and serotonin were observed in the sciatic nerve CCI rat model. There was a decrement in basal serotonin level within the ventrobasal thalamus of animals with neuropathic pain. ${ }^{48}$ Putative mechanisms mediating this change include alterations of the GABAergic system, ${ }^{48}$ and one can postulate that GABA and serotonin treatments may attenuate neuropathic pain based on these neurotransmitter results.

Activation of extrasynaptic GABA hyperpolarizes neurons and promotes burst firing, whereas blockage of these receptors promotes tonic over burst firing. ${ }^{49}$ However, other studies have suggested that tonic firing of thalamic relay cells is associated with nociceptive pain and burst firing is associated with the inhibition of nociceptive information processing. ${ }^{50,51}$ GABAergic transmission in the $\mathrm{ZI}$ is complex. Cha et al reported that muscimol injection into the ZI evoked ZI neuron inactivation, ${ }^{52,53}$ but the results of Moon et al did not agree. ${ }^{13}$ It would be possible to target on complex ZI itself and posterior thalmaus. Petronilho et $\mathrm{al}^{54}$ demonstrated that injection of glutamate into the ZI produced antinociception. They used an acute pain model to investigate short-duration antinociception after administration of glutamate, whereas a chronic neuropathic pain model was used in this study. They focused on a pain-inhibitory pathway that descends to the spinal cord via the dorsolateral funiculus using serotonergic, $\alpha$-adrenergic, and endogenous opioid-based mechanisms, whereas the focus here was on incerto-thalamic inhibition by GABAergic influences.

Although the cause of neuropathic pain remains unknown, it has been postulated that chronic neuropathic pain results from abnormally suppressed inhibition in the thalamus. Although GABAergic transmission is known to modulate states of arousal and pain, investigation of the underlying mechanisms is complicated because the involved region of the brain is extensive and GABAergic interneuron transmission is complex. The spinothalamic tract, which transmits signals that are important for pain localization, and the spinoreticular tract, which is involved in the emotional aspect of pain, are the two major pain pathways. The ZI receives dense nociceptive input through the spinothalamic tract ${ }^{55,56}$ and sends feed-forward tonic inhibition exclusively to thalamic nuclei, ${ }^{12,24,25}$ particularly the posterior nucleus of the thalamus. ${ }^{42,57}$ Neuropathic pain after CCI is thought to result from decreased neuronal activity in the ZI, which normally provides potent inhibition to select thalamic nuclei. Thus, the loss of ZI activity correlates with a pathological increase in neuronal activity in the posterior thalamus, a somatosensory thalamic nucleus critical for processing nociceptive information. ${ }^{12}$

\section{Limitations}

The results provide further evidence for a role of GABAergic signaling and neuronal activity in the ZI in neuropathic pain and related behavior. However, there are some limitations of this study that need to be considered. As infusion into the ZI can immediately affect sensitivity during pain behavior testing, changes in hypersensitivity were not measured during drug infusion.

Yalcin et $a 1^{58}$ suggested that 3 days of recovery are recommended to diminish post-surgery sensitivity, and the data tend to be consecutive. The size of the ZI can be very different in humans and rats; therefore, application to other species should be done with caution. This study did not perform GABA staining or count GABAergic neurons in CCI and sham-treated rats. However, using microdialysis, a reduction in GABA concentration was observed in CCI rats. Neuronal activity is influenced by the timing/status of anesthesia, and it is difficult to standardize the animals in this regard; therefore, observations were recorded within 1 hour of administering anesthesia. Monitoring freely moving animals is another 
technical approach, but this is difficult to apply to a pain model. Increased neuronal activity was also observed in the ZI after GABA agonist infusion in CCI rats, suggesting that neuronal activity in the ZI plays a role in neuropathic pain after CCI of the sciatic nerve.

\section{Conclusion}

The present study suggests that neuropathic pain in a rat sciatic nerve CCI model is caused by reduction of GABAergic signaling in the ZI. Furthermore, neurotransmitter analysis of CCI lesions appeared to reduce the GABA concentrations compared to sham-controlled rats. GABAergic drug into the ZI can ameliorate neuropathic pain in the rat sciatic CCI model. This implicates GABAergic drug modulating ZI, and therefore relieving neuropathic pain in vivo. The rat sciatic CCI induced hypo GABAergic state in ZI associated with thalamic firing that resulted in neuropathic pain.

\section{Acknowledgment}

This work was supported by the National Research Foundation of Korea (NRF 2014K1A3A1A21001372 and NRF2016H1D5A1908909).

\section{Disclosure}

The authors report no conflicts of interest in this work.

\section{References}

1. Jung BF, Ahrendt GM, Oaklander AL, Dworkin RH. Neuropathic pain following breast cancer surgery: proposed classification and research update. Pain. 2003;104(1-2):1-13.

2. Clark AK, Old EA, Malcangio M. Neuropathic pain and cytokines: current perspectives. J Pain Res. 2013;6:803-814.

3. Schmader KE. Epidemiology and impact on quality of life of postherpetic neuralgia and painful diabetic neuropathy. Clin J Pain. 2002; 18(6):350-354.

4. Raskin J, Pritchett YL, Wang F, et al. A double-blind, randomized multicenter trial comparing duloxetine with placebo in the management of diabetic peripheral neuropathic pain. Pain Med. 2005;6(5):346-356.

5. Heitmann H, Biberacher V, Tiemann L, et al. Prevalence of neuropathic pain in early multiple sclerosis. Mult Scler. 2016;22(9):1224-1230.

6. Torrance N, Smith BH, Bennett MI, Lee AJ. The epidemiology of chronic pain of predominantly neuropathic origin. Results from a general population survey. J Pain. 2006;7(4):281-289.

7. Bouhassira D, Lanteri-Minet M, Attal N, Laurent B, Touboul C. Prevalence of chronic pain with neuropathic characteristics in the general population. Pain. 2008;136(3):380-387.

8. Pereira EA, Aziz TZ. Neuropathic pain and deep brain stimulation. Neurotherapeutics. 2014;11(3):496-507.

9. Galluzzi KE. Managing neuropathic pain. J Am Osteopath Assoc. 2007;107(10 Suppl 6):ES39-ES48.

10. Austin PJ, Wu A, Moalem-Taylor G. Chronic constriction of the sciatic nerve and pain hypersensitivity testing in rats. JVis Exp. 2012;61:3393.

11. Boureau F, Doubrere JF, Luu M. Study of verbal description in neuropathic pain. Pain. 1990;42(2):145-152.

12. Masri R, Quiton RL, Lucas JM, Murray PD, Thompson SM, Keller A. Zona incerta: a role in central pain. JNeurophysiol. 2009;102(1):181-191.
13. Moon HC, Lee YJ, Cho CB, Park YS. Suppressed GABAergic signaling in the zona incerta causes neuropathic pain in a thoracic hemisection spinal cord injury rat model. Neurosci Lett. 2016;632:55-61.

14. Somers DL, Clemente FR. Dorsal horn synaptosomal content of aspartate, glutamate, glycine and GABA are differentially altered following chronic constriction injury to the rat sciatic nerve. Neurosci Lett. 2002;323(3):171-174.

15. Park A, Hoffman K, Keller A. Roles of GABAA and GABAB receptors in regulating thalamic activity by the zona incerta: a computational study. J Neurophysiol. 2014;112(10):2580-2596.

16. Vanini G, Lydic R, Baghdoyan HA. GABA-to-ACh ratio in basal forebrain and cerebral cortex varies significantly during sleep. Sleep. 2012;35(10):1325-1334.

17. Yousuf MS, Kerr BJ. The role of regulatory transporters in neuropathic pain. Adv Pharmacol. 2016;75:245-271.

18. Coull JA, Boudreau D, Bachand K, et al. Trans-synaptic shift in anion gradient in spinal lamina I neurons as a mechanism of neuropathic pain. Nature. 2003;424(6951):938-942.

19. Wardach J, Wagner M, Jeong Y, Holden JE. Lateral hypothalamic stimulation reduces hyperalgesia through spinally descending orexin-A neurons in neuropathic pain. West J Nurs Res. 2016;38(3):292-307.

20. Rusanescu G, Mao J. Peripheral nerve injury induces adult brain neurogenesis and remodelling. J Cell Mol Med. 2017;21(2):299-314.

21. Mitrofanis J. Some certainty for the "zone of uncertainty"? Exploring the function of the zona incerta. Neuroscience. 2005;130(1):1-15.

22. Shaw FZ, Liao YF, Chen RF, Huang YH, Lin RC. The zona incerta modulates spontaneous spike-wave discharges in the rat. J Neurophysiol. 2013;109(10):2505-2516.

23. Cavdar S, Onat F, Cakmak YO, Saka E, Yananli HR, Aker R. Connections of the zona incerta to the reticular nucleus of the thalamus in the rat. J Anat. 2006;209(2):251-258.

24. Trageser JC, Keller A. Reducing the uncertainty: gating of peripheral inputs by zona incerta. $J$ Neurosci. 2004;24(40):8911-8915.

25. Trageser JC, Burke KA, Masri R, Li Y, Sellers L, Keller A. Statedependent gating of sensory inputs by zona incerta. $J$ Neurophysiol. 2006;96(3):1456-1463.

26. Shaw FZ, Liao YF, Chen RF, Huang YH, Lin RC. The zona incerta modulates spontaneous spike-wave discharges in the rat. J Neurophysiol. 2013;109(10):2505-2516

27. Lee IO, Lim ES. Intracisternal or intrathecal glycine, taurine, or muscimol inhibit bicuculline-induced allodynia and thermal hyperalgesia in mice. Acta Pharmacol Sin. 2010;31(8):907-914.

28. Ran R, Gu J, Fu J, et al. The role of the GABA-A receptor of the adjacent intact dorsal root ganglion neurons in rats with neuropathic pain. Acta Neurobiol Exp. 2014;74(4):405-414.

29. Paxinos G, Watson C. The Rat Brain in Stereotaxic Coordinates. Sydney: Academic; 1986.

30. Mitsushima D, Mizuno T, Kimura F. Age-related changes in diurnal acetylcholine release in the prefrontal cortex of male rats as measured by microdialysis. Neuroscience. 1996;72(2):429-434.

31. Mitsushima D, Shwe TT, Funabashi T, Shinohara K, Kimura F. GABA release in the medial preoptic area of cyclic female rats. Neuroscience. 2002;113(1):109-114.

32. Tin Tin Win S, Mitsushima D, Shinohara K, Kimura F. Sexual dimorphism of GABA release in the medial preoptic area and luteinizing hormone release in gonadectomized estrogen-primed rats. Neuroscience. 2004;127(1):243-250.

33. Takase K, Mitsushima D, Masuda J, et al. Feeding with powdered diet after weaning affects sex difference in acetylcholine release in the hippocampus in rats. Neuroscience. 2005;136(2):593-599.

34. Azami J, Llewelyn MB, Roberts MH. The contribution of nucleus reticularis paragigantocellularis and nucleus raphe magnus to the analgesia produced by systemically administered morphine, investigated with the microinjection technique. Pain. 1982;12(3):229-246.

35. Abbasi S, Kumar SS. Electrophysiological and morphological characterization of cells in superficial layers of rat presubiculum. J Comp Neurol. 2013;521(13):3116-3132. 
36. Perera CJ, Duffy SS, Lees JG, et al. Active immunization with myelinderived altered peptide ligand reduces mechanical pain hypersensitivity following peripheral nerve injury. $J$ Neuroinflammation. 2015;12:28.

37. Ito A, Takeda M, Yoshimura T, et al. Anti-hyperalgesic effects of calcitonin on neuropathic pain interacting with its peripheral receptors. $\mathrm{Mol}$ Pain. 2012;8:42.

38. Wang X, Zhang Y, Kong L, et al. RSEP1 is a novel gene with functional involvement in neuropathic pain behaviour. Eur J Neurosci. 2005;22(5):1090-1096.

39. Diamond ME, Ahissar E. When outgoing and incoming signals meet: new insights from the zona incerta. Neuron. 2007;56(4):578-579.

40. Kita T, Osten P, Kita H. Rat subthalamic nucleus and zona incerta share extensively overlapped representations of cortical functional territories. J Comp Neurol. 2014;522(18):4043-4056.

41. Dubrovina NI, Il'iuchenok R. [Descending and ascending influences on zona incerta neurons]. Neirofiziologiia. 1980;12(1):46-52. Russian.

42. Bartho P, Freund TF, Acsady L. Selective GABAergic innervation of thalamic nuclei from zona incerta. Eur J Neurosci. 2002;16(6):999-1014

43. Jones EG. The Thalamus. Cambridge: Cambridge University Press 2007.

44. Gwak YS, Crown ED, Unabia GC, Hulsebosch CE. Propentofylline attenuates allodynia, glial activation and modulates GABAergic tone after spinal cord injury in the rat. Pain. 2008;138(2):410-422.

45. Drew GM, Siddall PJ, Duggan AW. Mechanical allodynia following contusion injury of the rat spinal cord is associated with loss of GABAergic inhibition in the dorsal horn. Pain. 2004;109(3):379-388.

46. Hains BC, Waxman SG. Activated microglia contribute to the maintenance of chronic pain after spinal cord injury. $J$ Neurosci. 2006;26(16):4308-4317.

47. Iura A, Takahashi A, Hakata S, Mashimo T, Fujino Y. Reductions in tonic GABAergic current in substantia gelatinosa neurons and GABAA receptor $\delta$ subunit expression after chronic constriction injury of the sciatic nerve in mice. Eur J Pain. 2016;20(10):1678-1688.
48. Goettl VM, Huang Y, Hackshaw KV, Stephens RL Jr. Reduced basal release of serotonin from the ventrobasal thalamus of the rat in a model of neuropathic pain. Pain. 2002;99(1-2):359-366.

49. Cope DW, Hughes SW, Crunelli V. GABAA receptor-mediated tonic inhibition in thalamic neurons. $J$ Neurosci. 2005;25(50):11553-11563.

50. Cheong E, Lee S, Choi BJ, Sun M, Lee CJ, Shin HS. Tuning thalamic firing modes via simultaneous modulation of T- and L-type $\mathrm{Ca} 2+$ channels controls pain sensory gating in the thalamus. $J$ Neurosci. 2008;28(49):13331-13340.

51. Kim D, Park D, Choi S, et al. Thalamic control of visceral nociception mediated by T-type Ca2+ channels. Science. 2003;302(5642): 117-119.

52. Cha M, Ji Y, Masri R. Motor cortex stimulation activates the incertothalamic pathway in an animal model of spinal cord injury. J Pain. 2013;14(3):260-269.

53. Lucas JM, Ji Y, Masri R. Motor cortex stimulation reduces hyperalgesia in an animal model of central pain. Pain. 2011;152(6):1398-1407.

54. Petronilho A, Reis GM, Dias QM, Fais RS, Prado WA. Antinociceptive effect of stimulating the zona incerta with glutamate in rats. Pharmacol Biochem Behav. 2012;101(3):360-368.

55. Craig AD. Distribution of trigeminothalamic and spinothalamic lamina I terminations in the macaque monkey. J Comp Neurol. 2004;477(2):119-148.

56. Shammah-Lagnado SJ, Negrao N, Ricardo JA. Afferent connections of the zona incerta: a horseradish peroxidase study in the rat. Neuroscience. 1985;15(1):109-134.

57. Power BD, Kolmac CI, Mitrofanis J. Evidence for a large projection from the zona incerta to the dorsal thalamus. J Comp Neurol. 1999; 404(4):554-565.

58. Yalcin I, Megat S, Barthas F, et al. The sciatic nerve cuffing model of neuropathic pain in mice. J Vis Exp. 2014;89:51608. 


\section{Supplementary material}

Table SI Firing rate in zona incerta

\begin{tabular}{|c|c|c|c|c|c|c|}
\hline \multirow[t]{3}{*}{ Monitoring periods } & \multicolumn{6}{|c|}{ Firing rate (spikes/s) } \\
\hline & \multicolumn{2}{|l|}{ Saline injection } & \multicolumn{2}{|c|}{ GABA agonist injection } & \multicolumn{2}{|c|}{ GABA antagonist injection } \\
\hline & Sham-treated & $\mathrm{CCl}$ & Sham-treated & $\mathbf{C C l}$ & Sham-treated & $\mathrm{CCl}$ \\
\hline Baseline (-10 to $0 \mathrm{~min})$ & $7.42 \pm 0.25$ & $2.45 \pm 0.27$ & $7.56 \pm 0.16$ & $5.52 \pm 0.17$ & $10.16 \pm 0.25$ & $4.31 \pm 0.14$ \\
\hline Portion I (0-10 min) & $4.51 \pm 0.15$ & $3.5 I \pm 0.3 \mathrm{I}$ & $21.18 \pm 0.22$ & $31.8 \pm 1.19$ & $34.91 \pm 0.29$ & $5.02 \pm 0.17$ \\
\hline Portion II (I0-20 min) & $7.02 \pm 0.27$ & $6.57 \pm 0.30$ & $26.78 \pm 0.84$ & $35.72 \pm 1.27$ & $40.37 \pm 0.97$ & $5.34 \pm 0.16$ \\
\hline Portion III (20-30 min) & $11.99 \pm 0.29$ & $8.00 \pm 0.27$ & $26.6 \pm 0.25$ & $50.65 \pm 2.04$ & $26.6 \pm 0.25$ & $5.36 \pm 0.14$ \\
\hline
\end{tabular}

Notes: Baseline is the spontaneous neuronal activity before injection. Neuronal activity is divided by time. Firing rate is defined as spikes per second. Values represent mean \pm SED.

Abbreviations: $\mathrm{CCl}$, chronic constriction injury; GABA, gamma-aminobutyric acid; SED, standard error of the mean.

The Journal of Pain Research is an international, peer reviewed, open access, online journal that welcomes laboratory and clinical findings in the fields of pain research and the prevention and management of pain. Original research, reviews, symposium reports, hypothesis formation and commentaries are all considered for publication.

\section{Dovepress}

The manuscript management system is completely online and includes a very quick and fair peer-review system, which is all easy to use. Visit http://www.dovepress.com/testimonials.php to read real quotes from published authors. 